\title{
Reduced Prefrontal Connectivity in Psychopathy
}

\author{
Julian C. Motzkin, ${ }^{1}$ Joseph P. Newman, ${ }^{2}$ Kent A. Kiehl, ${ }^{3,4}$ and Michael Koenigs ${ }^{1}$ \\ ${ }^{1}$ Department of Psychiatry, University of Wisconsin-Madison, Madison, Wisconsin 53719, ${ }^{2}$ Department of Psychology, University of Wisconsin-Madison, \\ Madison, Wisconsin 53706, ${ }^{3}$ The MIND Research Network, Albuquerque, New Mexico 87131, and ${ }^{4}$ Departments of Psychology and Neuroscience, \\ University of New Mexico, Albuquerque, New Mexico 87131
}

Linking psychopathy to a specific brain abnormality could have significant clinical, legal, and scientific implications. Theories on the neurobiological basis of the disorder typically propose dysfunction in a circuit involving ventromedial prefrontal cortex (vmPFC). However, to date there is limited brain imaging data to directly test whether psychopathy may indeed be associated with any structural or functional abnormality within this brain area. In this study, we employ two complementary imaging techniques to assess the structural and functional connectivity of vmPFC in psychopathic and non-psychopathic criminals. Using diffusion tensor imaging, we show that psychopathy is associated with reduced structural integrity in the right uncinate fasciculus, the primary white matter connection between vmPFC and anterior temporal lobe. Using functional magnetic resonance imaging, we show that psychopathy is associated with reduced functional connectivity between vmPFC and amygdala as well as between vmPFC and medial parietal cortex. Together, these data converge to implicate diminished vmPFC connectivity as a characteristic neurobiological feature of psychopathy.

\section{Introduction}

Psychopathy is a neuropsychiatric disorder that imposes a substantial burden on society. Typified by callous and impulsive antisocial behavior, psychopathy is present in approximately a quarter of adult prison inmates, and it is associated with a disproportionately high incidence of violent crime and recidivism (Hare, 2003). The identification of neural correlates of the disorder could thus have profound implications for the clinical and legal management of psychopathic criminals, as well as for the basic understanding of the biological substrates underlying human social behavior. However, consistent and replicable neural correlates of psychopathy have remained elusive, due in large part to the methodological and logistical challenges associated with using neuroimaging to study this population (Koenigs et al., 2011a).

Systems-level neurobiological models of psychopathy have focused primarily on limbic and paralimbic structures (Kiehl, 2006) involved in the regulation of emotion and social behavior, particularly the amygdala and ventromedial prefrontal cortex (vmPFC) (Blair, 2007, 2008). Perhaps the most compelling support for a central role of vmPFC dysfunction in psychopathy has emerged from the study of neurological patients with focal vmPFC lesions. For decades, neurologists have noted that the

\footnotetext{
Received Aug. 15, 2011; revised Sept. 21, 2011; accepted Oct. 10, 2011.

Author contributions: J.P.N., K.A.K., and M.K. designed research; J.C.M. and M.K. analyzed data; J.C.M. and M.K. wrote the paper.

This work was supported by a University of Wisconsin (UW)-Madison/UW-Milwaukee Intercampus Research Incentive Grant and grants from the National Institutes of Health (MH070539, DA026505, MH086787, T32MH018931-21, and T32GM007507). We thank Keith Harenski for his assistance with MRI data collection and C.P. Frost for his assistance with amygdala tracing. We thank many at the Wisconsin Department of Corrections for making this research possible.

Correspondence should be addressed to Michael Koenigs, Department of Psychiatry, University of WisconsinMadison, 6001 Research Park Boulevard, Madison, WI 53719. E-mail: mrkoenigs@wisc.edu.

DOI:10.1523/JNEUROSCI.4215-11.2011

Copyright $\odot 2011$ the authors $\quad 0270-6474 / 11 / 3117348-10 \$ 15.00 / 0$
}

personality changes accompanying vmPFC damage (e.g., lack of empathy, irresponsibility, and poor decision making) bear striking resemblance to hallmark psychopathic personality traits. Indeed, the personality changes associated with vmPFC damage have been dubbed "pseudopsychopathy" (Blumer and Benson, 1975) and "acquired sociopathy" (Eslinger and Damasio, 1985). In recent studies, we have addressed this similarity experimentally. Using laboratory decision-making tests, we found that psychopaths - particularly the low-anxious or "primary" subtypeperform remarkably similar to vmPFC lesion patients on measures of social economic choice (Koenigs et al., 2010) and moral judgment (Koenigs et al., 2011b). Together, these data suggest that vmPFC dysfunction may be a critical neurobiological substrate underlying psychopathy.

Of course, the hypothesized role of vmPFC in psychopathy depends on the reciprocal interactions between vmPFC and other cortical and subcortical brain regions involved in affective processing, social cognition, and decision making. Two areas of particular interest in this regard are the amygdala and a medial parietal area including the precuneus and posterior cingulate cortex (PCC). Both the amygdala and precuneus/PCC are densely and reciprocally connected with vmPFC (Price, 1999; Barbas, 2000; Raichle et al., 2001; Greicius et al., 2003), and both areas have been associated with reduced activity in psychopathy (Kiehl et al., 2001; Glenn et al., 2009). Moreover, the interactions between vmPFC and these areas are thought to subserve key functions related to psychopathy. vmPFC-amygdala interactions are thought to underlie aspects of emotion regulation, aggression, and stimulus-reinforcement associations (Davidson et al., 2000; Milad et al., 2006; Blair, 2008; Delgado et al., 2008), while vmPFC-precuneus/PCC interactions are thought to underlie aspects of self-reflective processing (Buckner et al., 2008; Qin and Northoff, 2011). Therefore, investigating the structural and functional connectivity between vmPFC and these brain regions will 
be a key step toward understanding the disordered neural interactions that contribute to psychopathic behavior.

In the present study, we employ two complementary neuroimaging methods to quantify the structural and functional connectivity of vmPFC in psychopathic and non-psychopathic prison inmates. Using diffusion tensor imaging (DTI), we test the hypothesis that psychopathy is associated with reduced structural integrity of the uncinate fasciculus (UF), the primary white matter pathway connecting vmPFC with anterior temporal lobe structures, including amygdala. Using functional magnetic resonance imaging (fMRI), we test the hypothesis that psychopathy is associated with lower levels of correlated activity between vmPFC and interconnected brain structures (amygdala and medial parietal cortex) at rest. For both imaging measures, we conduct follow-up analyses to determine whether any of the observed neurobiological differences between psychopaths and nonpsychopaths are driven by a particular subtype of psychopathy (low-anxious or "primary" vs high-anxious or "secondary").

\section{Materials and Methods}

\section{Participants}

Participants were adult male inmates recruited from a medium-security Wisconsin correctional institution. Inmates were eligible if they met the following criteria: $<45$ years of age, IQ $>70$, no history of psychosis or bipolar disorder, and not currently taking psychotropic medications. Informed consent was obtained both orally and in writing.

The Psychopathy Checklist-Revised (PCL-R) (Hare, 2003) was used to assess psychopathy. The PCL-R assessment involves a 60-90 min interview and file review to obtain information used to rate 20 psychopathyrelated items as 0,1 , or 2 , depending on the degree to which each trait characterizes the individual. A substantial literature supports the reliability and validity of PCL-R assessments with incarcerated offenders (Hare, 2003). To evaluate interrater reliability, a second rater who was present during interviews provided independent PCL-R ratings for eight inmates. The intraclass correlation coefficient was 0.85 . PCL-R Factor 1 and 2 scores were computed following procedures outlined in the PCL-R manual (Hare, 2003).

\section{Participant groups}

Participants were classified as psychopathic if their PCL-R scores were 30 or greater and non-psychopathic if their PCL-R scores were 20 or less (Hare, 2003). (The use of these cut scores affords clear distinction between high and low levels of psychopathy, but precludes the correlation of imaging data with PCL-R factor or facet scores, which would require a more continuous range of PCL-R scores.) Following the convention of previous studies identifying psychopathic subtypes (Arnett et al., 1997; Lorenz and Newman, 2002; Brinkley et al., 2004; Hiatt et al., 2004; Koenigs et al., 2010), psychopaths were subdivided based on a median split of Welsh Anxiety Scale (WAS) scores (Welsh, 1956). Thus, in our sample low-anxious psychopathy was defined as having a PCL-R score of 30 or greater and a WAS score of 13 or less, while high-anxious psychopathy was defined as having a PCL-R score of 30 or greater and a WAS score of 14 or greater.

\section{MRI data collection}

All MR imaging data were acquired using the Mind Research Network's mobile Siemens 1.5 T Avanto Mobile MRI System with advanced SQ gradients (max slew rate $200 \mathrm{~T} / \mathrm{m} / \mathrm{s}, 346 \mathrm{~T} / \mathrm{m} / \mathrm{s}$ vector summation, rise time $200 \mu$ s) equipped with a 12 -element head coil. Head motion was limited using padding and restraint. All prisoners were scanned on correctional facility grounds.

\section{DTI study}

DTI participants. A total of 27 inmates $(n=14$ psychopaths and $n=13$ non-psychopaths) meeting the inclusion criteria participated in the DTI study (Table 1).

DTI data collection. Diffusion-weighted echo-planar magnetic resonance images were acquired by applying diffusion sensitizing gradients along 30 non-collinear directions $\left(b\right.$ value $\left.=800 \mathrm{~s} / \mathrm{mm}^{2}\right)$. Five interleaved non-diffusion-weighted ( $b$ value $=0 \mathrm{~s} / \mathrm{mm}^{2}$ ) volumes were collected during each run to enable corrections for motion and eddy current distortions. Images were collected with the following parameters: repetition time $(\mathrm{TR})=9200 \mathrm{~ms}$, echo time $(\mathrm{TE})=84 \mathrm{~ms}$, field of view $(\mathrm{FOV})=256 \mathrm{~mm} \times 256 \mathrm{~mm}$, matrix size $=128 \times 128$, slice thickness $=$ $2 \mathrm{~mm}$, no gap, voxel size $=2 \mathrm{~mm} \times 2 \mathrm{~mm} \times 2 \mathrm{~mm}$, 70 slices. The sequence was repeated twice and the data combined to improve SNR.

Data processing was conducted using the FSL (http://www.fmrib.ox. ac.uk/fsl/) and CAMINO (Cook et al., 2006) software packages. Eddy current distortions and head movements were corrected by affine registration of all images to the first non-diffusion-weighted volume (FSL). Nonbrain tissue was removed using the brain extraction toolbox (BET) in FSL (Smith, 2002), and the resulting brain masks were carefully inspected before performing nonlinear diffusion tensor estimation using CAMINO (Jones and Basser, 2004; Alexander and Barker, 2005). Diffusion tensor images for each subject were screened for extreme outlier voxels and resampled to $1.75 \mathrm{~mm} \times 1.75 \mathrm{~mm} \times 2.5 \mathrm{~mm}$ resolution. Fractional anisotropy (FA), a scalar measure of fiber coherence and microstructural white matter integrity, was calculated from the diffusion tensors at each brain voxel.

FA maps for each subject were nonlinearly registered to the standard MNI152 white matter template and resampled to $1 \mathrm{~mm}^{3}$ resolution (Andersson et al., 2007). Aligned images were averaged into a group mean FA image, which was thinned to create a study-specific white matter skeleton mask. This mask was projected onto each subject's native space FA image and whole-brain estimates were acquired by extracting the mean FA value across the white matter skeleton. Group differences were examined in the UF, the primary structural connection between the amygdala and vmPFC, and in comparison tracts with documented frontal and temporal connectivity, in which no group differences were expected (inferior longitudinal fasciculus/inferior fronto-occipital fasciculus, ILF/IFOF; superior longitudinal fasciculus, SLF; superior fronto-occipital fasciculus, SFOF). Individual FA estimates for each region of interest (ROI) were acquired by projecting the Johns Hopkins University (JHU) white matter atlas onto each subject's native space FA image and computing the mean value across the tract labels of interest (Mori et al., 2005). FA values for each ROI were scaled to whole-brain FA and entered into two-sample $t$ tests to assess group differences between psychopaths and nonpsychopaths. To determine the effects of psychopathy subtype (primary, "low-anxious" and secondary, "high-anxious") on structural connectivity, mean scaled FA estimates from the right UF were entered into nonparametric Mann-Whitney $U$ tests. All DTI analyses were considered significant at $p<0.05$.

To confirm the anatomical validity of UF ROIs used in the betweengroups analysis, UF tracts were verified in every subject using tractography. Briefly, fiber tracking was performed from every voxel in the brain ("brute force" method) using the tensor deflection (TEND) algorithm in CAMINO (Lazar et al., 2003). Individual UF tracts were isolated by filtering tractography results to include only those tracts that passed through the UF ROI defined by the JHU atlas (Fig. 1a). Tracts acquired in this way were compared to tracts derived using the manual two-ROI approach, a technique shown to reliably identify tracts with the characteristic "C" shape of UF fibers (Wakana et al., 2007; Hua et al., 2008). Fiber trajectories for each subject were confirmed using known neuroanatomical landmarks and white matter atlases to verify that the ROIs sampled from white matter regions containing predominantly UF fibers. All tractography data were processed and analyzed using TrackVis software (Wang et al., 2007).

\section{Rest-fMRI study}

Rest-fMRI participants. A total of 40 inmates ( $n=20$ psychopaths and $n=20$ non-psychopaths) meeting the inclusion criteria participated in the rest-fMRI study (Table 1). Twenty-six inmates had both DTI and rest-fMRI data.

Rest-fMRI data collection. Resting state functional images were collected while subjects lay still and awake, passively viewing a fixation cross. $\mathrm{T} 2{ }^{\star}$-weighted gradient-echo echoplanar functional images (EPIs) were acquired with the following parameters: TR $=2000 \mathrm{~ms}$, TE $=39 \mathrm{~ms}$, flip 
Table 1. Participant group characteristics

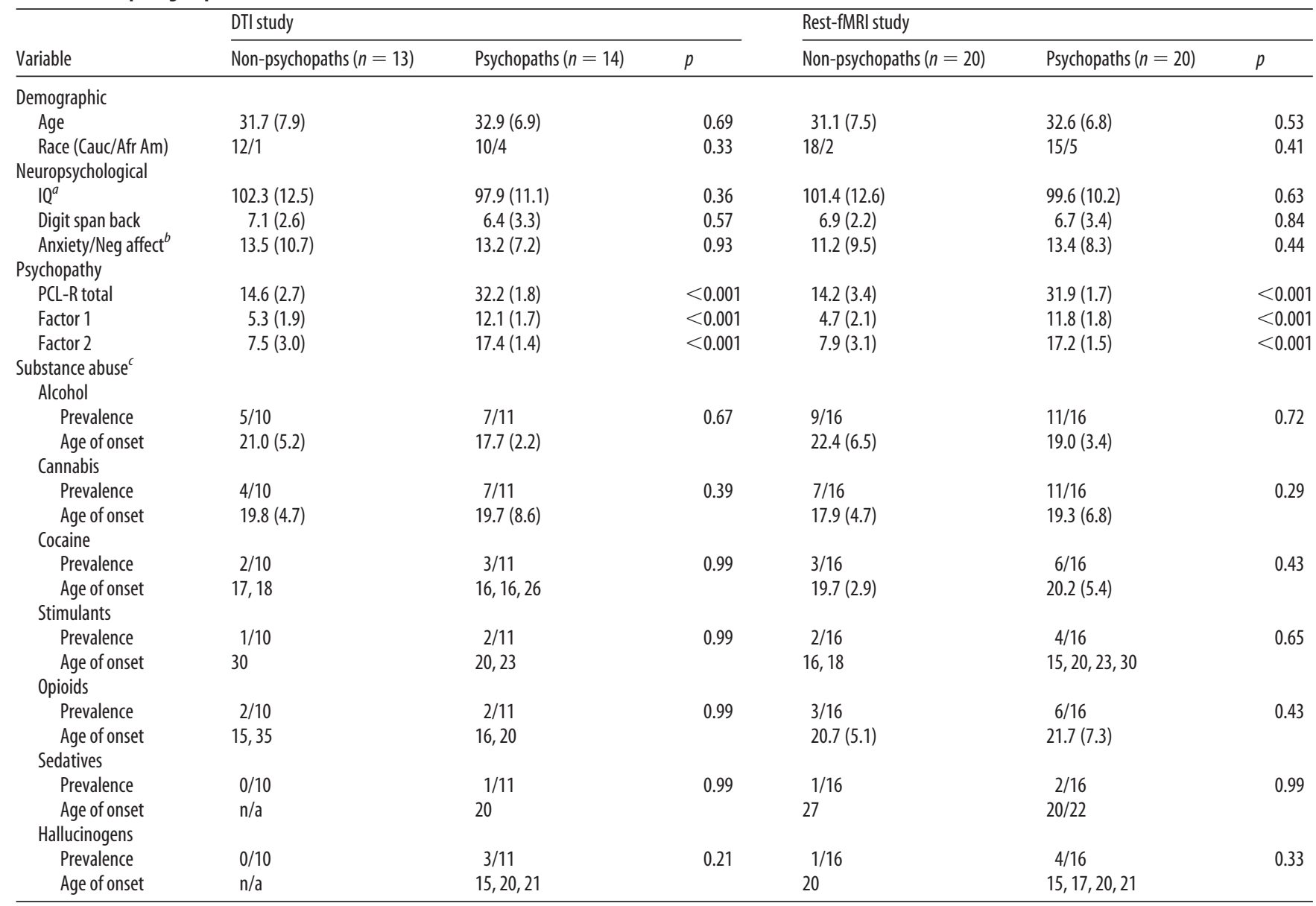

${ }^{a}$ Based on Shipley Institute of Living Scale (Zachary, 1986), ${ }^{b}$ based on Welsh Anxiety Scale, 'based on diagnosis of abuse or dependence in the Structured Clinical Interview for DSM-IV Disorders (SCID) (First, 2002), which was administered to 10/13 non-psychopaths and 11/14 psychopaths in the DTI study and 16/20 non-psychopaths and 16/20 psychopaths in the rest-fMRI study. p values for race distribution and substance abuse prevalence were computed with Fisher's exact test. All other $p$ values are based on $t$ test (means presented followed by SDs in parentheses). $p$ values were not calculated for substance abuse age of onset due to relatively small sample sizes of abusers for most substances.

angle $=75^{\circ}, \mathrm{FOV}=24 \times 24 \mathrm{~cm}$, matrix $=64 \times 64$, slice thickness $=4$ $\mathrm{mm}$, gap $=1 \mathrm{~mm}$, voxel size $=3.75 \mathrm{~mm} \times 3.75 \mathrm{~mm} \times 5 \mathrm{~mm}, 27$ sequential axial oblique slices. Resting-state scans lasted $5.5 \mathrm{~min}$ (158 volumes). A high-resolution T1-weighted structural image was acquired using a four-echo MPRAGE sequence $(\mathrm{TR}=2530 ; \mathrm{TE}=1.64,3.5,5.36$, $7.22 \mathrm{~ms}$; flip angle $=7^{\circ}, \mathrm{FOV}=256 \times 256 \mathrm{~mm}$, matrix $=128 \times 128$, slice thickness $=1.33 \mathrm{~mm}$, no gap, voxel size $=1 \mathrm{~mm} \times 1 \mathrm{~mm} \times 1.33 \mathrm{~mm}$, 128 interleaved sagittal slices). All four echoes were averaged into a single high-resolution image, which was used to aid in the spatial normalization of EPI volumes and visualization of group statistics.

Preprocessing. All fMRI data analysis was performed using AFNI (Cox, 1996) and FSL (http://www.fmrib.ox.ac.uk/fsl/). EPI volumes were slice time corrected using the first slice as a reference (sequential acquisition, Fourier interpolation) and motion corrected by rigid body alignment to the first EPI acquisition. Any subject with motion $>4 \mathrm{~mm}$ in any direction was excluded from further analysis. The first three volumes were omitted from the EPI time series and the data were despiked to remove extreme time series outliers. Time series data were bandpass filtered $(0.009<f<0.08)$ before spatially smoothing with a $4 \mathrm{~mm}$ full-width at half-maximum Gaussian kernel. EPI time series data and high-resolution T1 images were normalized to the Talairach coordinate system (Talairach and Tournoux, 1988) using a 12-parameter linear warp and the EPI data were resampled to $3 \mathrm{~mm}$ cubic voxels for subsequent functional connectivity analyses. Normalized T1 anatomical images were segmented into gray matter, white matter, and CSF segments using FAST in FSL (Zhang et al., 2001). White matter and CSF segments were used as masks to extract a representative time series from each tissue type.

ROI selection and correlation analysis. To investigate differences in vmPFC connectivity between psychopaths and non-psychopaths, we ex- amined two functional networks with documented vmPFC involvement. To test the hypothesis that psychopathy is associated with reduced vmPFC-amygdala connectivity, we seeded the right and left amygdala in each subject. Amygdala ROIs were manually traced on the Talairachaligned group average T1 anatomical image (Nacewicz et al., 2006) and subsequently edited by cycling through each subject's T1 image, excluding voxels in which the group mask overlapped with adjacent, nonamygdalar, tissue (Fig. $2 a$ ). The final exclusionary mask contained only voxels overlying the amygdala in all subjects. To test the hypothesis that psychopathy is associated with reduced vmPFC-medial parietal connectivity, we seeded the precuneus/PCC region (Fig. $3 b$ ) using the coordinates reported by Fox et al. (2005) ( $-5,-49,40 ; 6$-mm-radius sphere).

Functional connectivity was assessed by computing whole-brain correlations with the mean time series derived from each of the seed ROIs. The mean time series was included in a GLM with eight regressors of no interest, including six motion parameters (three translations, three rotations) obtained from the rigid body alignment of EPI volumes, the ventricular time series acquired by averaging across the CSF mask, the white matter time series acquired by averaging across the white matter mask, and a second-order polynomial to model baseline signal and slow drift. Voxelwise correlation coefficients for each ROI were converted to $z$ scores via Fisher's $r$ to $z$ transform and the resulting $z$-score maps were entered into second-level statistical analyses.

Statistical analyses of correlation maps. To investigate differences in functional connectivity between psychopathic and non-psychopathic prisoners, we performed unpaired two-sample $t$ tests on the $z$-score maps derived from each seed region of interest. Based on our a priori interest in connectivity with the vmPFC, we restricted group comparisons to a region encompassing the medial surface of the PFC bilaterally, ventral to 


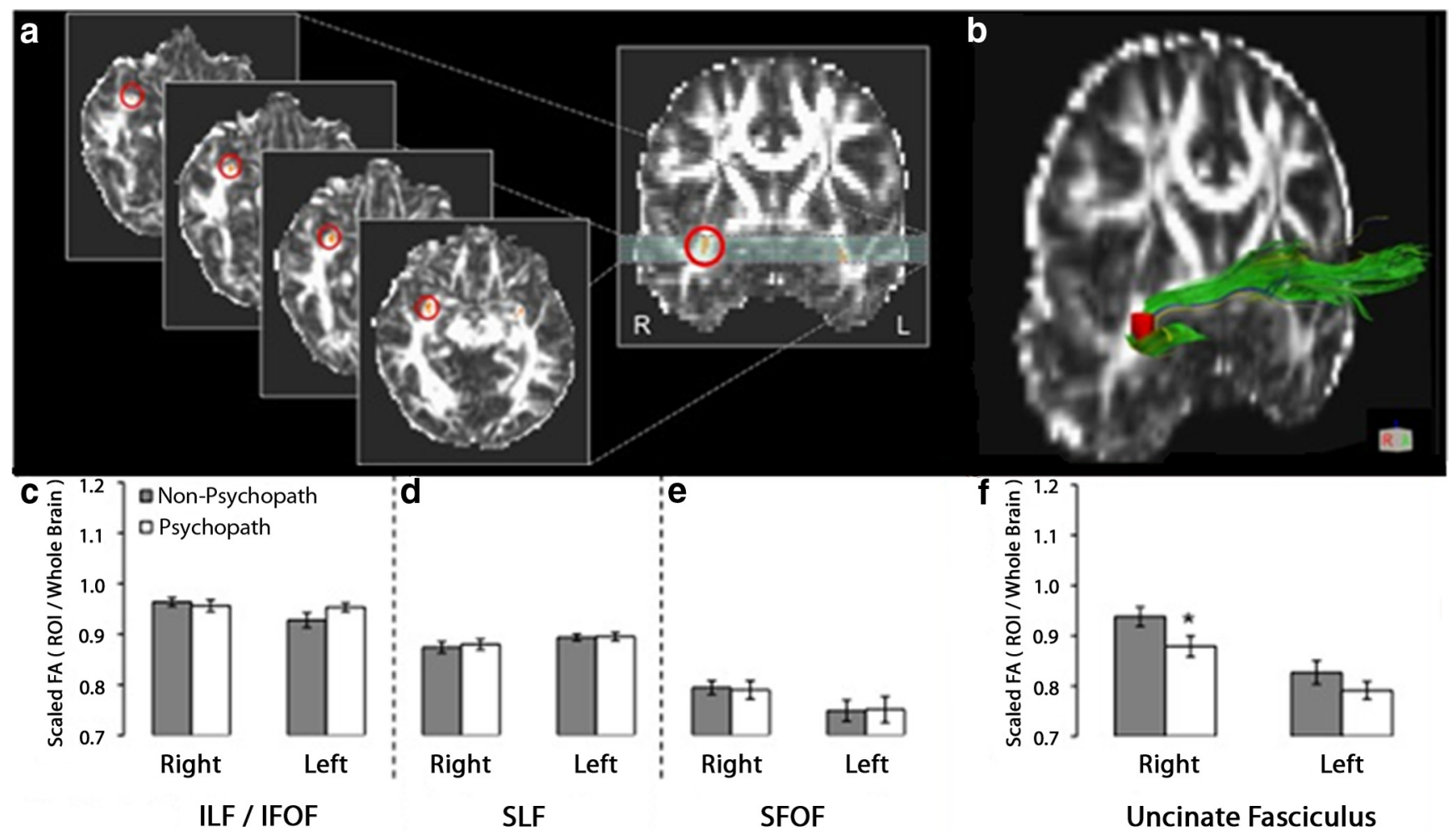

Figure 1. DTI results: reduced white matter integrity is specific to the right UF in psychopaths. $\boldsymbol{a}$, The UF ROI (circled in red) in serial axial slices and a single coronal slice. $\boldsymbol{b}$, The UF ROI (red) superimposed on an entire UF tract, as computed with tractography (see Materials and Methods for additional details). c $\boldsymbol{e}$, Bar plots of mean scaled FA values in three comparison tracts, in each hemisphere: ILF/IFOF (c), SLF (d), and SFOF (e). f, Bar plots of mean scaled FA values in the UF. Psychopaths exhibited significantly lower scaled FA values only in right UF. Error bars indicate SEM. ${ }^{*} p<0.05$.

the genu of the corpus callosum $(z=-6.5$; Figs. $2 a, 3 b)$. The vmPFC ROI was created using the WFU PickAtlas (Maldjian et al., 2003) and subsequently edited in AFNI to exclude regions dorsal to the genu. Group difference maps were corrected for multiple comparisons using clusterextent thresholding at an uncorrected $p<0.005, \alpha=0.05$ (cluster size $=$ 14 voxels, $378 \mathrm{~mm}^{3}$ ). Cluster extents were computed using Monte Carlo simulations implemented in the 3dClustSim program (AFNI). Group correlation maps were overlaid on the normalized mean anatomical image. All coordinates are reported in Talairach space.

To assess whether group differences were specific to vmPFC, we examined connectivity in two additional regions per seed in which we expected no group differences in functional connectivity. For the right amygdala seed, we investigated connectivity with the anterior superior temporal gyrus (aSTG) and contralateral amygdala, regions with documented right amygdala connectivity (Roy et al., 2009). Connectivity with the aSTG was assessed by placing a sphere of $6 \mathrm{~mm}$ radius at coordinates reported by Roy et al. (2009) (aSTG: 34, -10, -26). Connectivity with the left amygdala was assessed using the hand-drawn amygdala ROI from the previous connectivity analysis. For the precuneus/PCC seed, connectivity was assessed in bilateral parahippocampal gyrus and inferior parietal lobule (IPL) masks created in using the WFU PickAtlas (Maldjian et al., 2003). Group differences were considered significant at a corrected $p<0.05$ (uncorrected $p<0.005$, cluster size $=14$ voxels, $378 \mathrm{~mm}^{3}$ ).

To determine the effects of psychopathy subtype on resting connectivity, masks were generated from significant clusters and parameter estimates were extracted for each subject. These estimates were entered into a $2 \times 2$ ANOVA with anxiety and psychopathy as between-subjects factors.

\section{Results}

Structural connectivity: DTI results

FA is a scalar measure that is sensitive to the microstructural integrity and fiber coherence of white matter tracts (Pierpaoli and Basser, 1996). Here, we used FA to probe differences in white matter structural connectivity between psychopathic and nonpsychopathic prisoners. Before examining FA values in the UF, we first computed a measure of whole-brain FA for each subject, equal to the average FA within all major white matter tracts. Overall, psychopaths had significantly lower whole-brain FA than non-psychopaths $(t=-3.5, p=0.002)$. Therefore, in subsequent analyses of specific tracts, we computed a scaled FA value, equal to the FA value in the specific tract of interest divided by the subject's whole-brain FA value. Using the scaled FA values, we addressed our first hypothesis - that psychopaths would have reduced structural integrity (lower FA values) in the UF. Indeed, relative to non-psychopaths, psychopaths had significantly lower scaled FA values in the right UF $(t=-2.1, p=0.048$ ) (Fig. $1 a, b, f)$. To assess the anatomical specificity of this finding, we computed scaled FA values for three additional major fiber tracts involving the frontal lobe: the superior fronto-occipital fasciculus, the superior longitudinal fasciculus, and the inferior longitudinal fasciculus/inferior fronto-occipital fasciculus. In none of these comparison tracts were FA values significantly different between psychopaths and non-psychopaths (all $p$ values $>0.14$ ) (Fig. $1 c-e$ ). These DTI data associate psychopathy with a reduction of white matter integrity in the right UF.

\section{Functional connectivity: rest-fMRI results}

To assess the resting functional connectivity of vmPFC, we defined two "seed" regions known to be functionally interconnected with vmPFC at rest: amygdala (Roy et al., 2009; Kim et al., 2011) and precuneus/PCC (Greicius et al., 2003; Fox et al., 2005).

In light of our DTI findings in the right UF, we first examined resting functional connectivity between the right amygdala and vmPFC (Fig. 2a). For each group (psychopaths and non- 


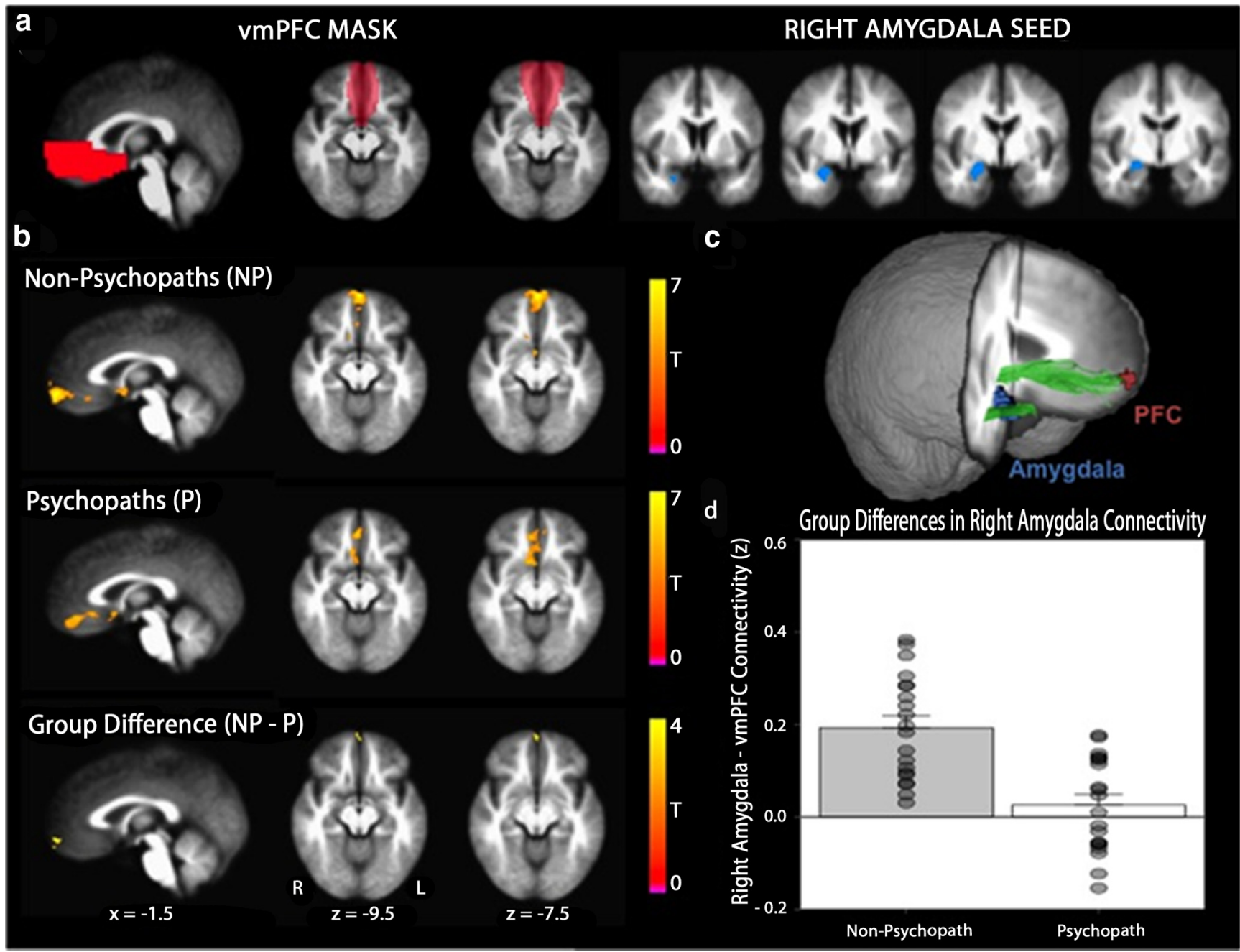

Figure 2. Functional connectivity between the right amygdala and anterior vmPFC is reduced in psychopaths. $\boldsymbol{a}$, Group differences in connectivity were assessed in the vmPFC mask (red) for correlation coefficients computed using the mean time series extracted from the hand drawn right amygdala seed (blue). $\boldsymbol{b}$, Mean right amygdala-vmPFC connectivity maps for non-psychopaths and psychopaths are shown separately on the group mean anatomical image, thresholded at a cluster corrected $p<0.05$. Scale bars depict the uncorrected $t$ statistic. Both groups exhibit significant resting connectivity between right amygdala and regions of vmPFC. The group difference map indicates an area in anterior vmPFC where non-psychopaths have significantly greater connectivity than psychopaths $(x=-3, y=-66, z=-10$, cluster size $=14$ voxels). $c$, A three-dimensional rendering of the group mean anatomical image shows the location of the amygdala seed (blue) and significant vmPFC cluster (red) in relation to the UF (green). $\boldsymbol{d}$, The bar plot depicts the significant group difference in connectivity estimates (Fisher z-transformed correlation coefficients) within the vmPFC cluster. Error bars indicate SEM. Filled circles represent values from individual subjects.

psychopaths), we computed the spatial map of voxels in vmPFC exhibiting a significant correlation with the BOLD signal derived from the right amygdala seed region (Fig. $2 b$ ). To test the hypothesis that psychopaths would have reduced vmPFC-amygdala functional connectivity at rest, we then computed the spatial map of vmPFC voxels with significant differences in correlated activity with right amygdala (Fig. $2 b$ ). In support of our hypothesis, we found a cluster of voxels in the right anterior vmPFC exhibiting significantly lower correlation with right amygdala in psychopaths than in non-psychopaths.

To address the anatomical specificity of this finding, we also assessed resting connectivity between the amygdala seed region and two other areas of the brain that have previously been shown to be functionally connected with the right amygdala: the contralateral (left) amygdala and a region of right aSTG (Roy et al., 2009). Although there were significant BOLD correlations between right and left amygdala as well as between right amygdala and right aSTG in both psychopaths and non-psychopaths, there were no significant between-group differences in amygdala connectivity with either area (both $p$ values $>0.17$ ). In other words, psychopaths exhibited normal functional connectivity between right amygdala and left amygdala, as well as between right amygdala and right aSTG. These fMRI results associate psychopathy with a reduction in vmPFC-amygdala functional connectivity.

To examine whether psychopaths also exhibit reduced functional connectivity between vmPFC and cortical brain regions, we computed resting BOLD correlations with a seed region located in right precuneus/PCC (Fig. $3 b$ ). The precuneus/PCC and vmPFC are known to exhibit significant correlations in BOLD signal at rest; together, they comprise the major midline structures of the "default mode network" (Shulman et al., 1997; Raichle et al., 2001; Greicius et al., 2003; Fox et al., 2005). Indeed, in our sample, both subject groups (psychopaths and nonpsychopaths) demonstrated significant correlations between precuneus/PCC and areas within vmPFC (Fig. 3a). In the betweengroup comparison, we again found a cluster of voxels in the right anterior vmPFC exhibiting significantly lower correlation with right precuneus/PCC in psychopaths than in non-psychopaths, as well as a cluster in more posterior vmPFC overlapping the rostral anterior cingulate cortex (rACC) (Fig. 3a). 


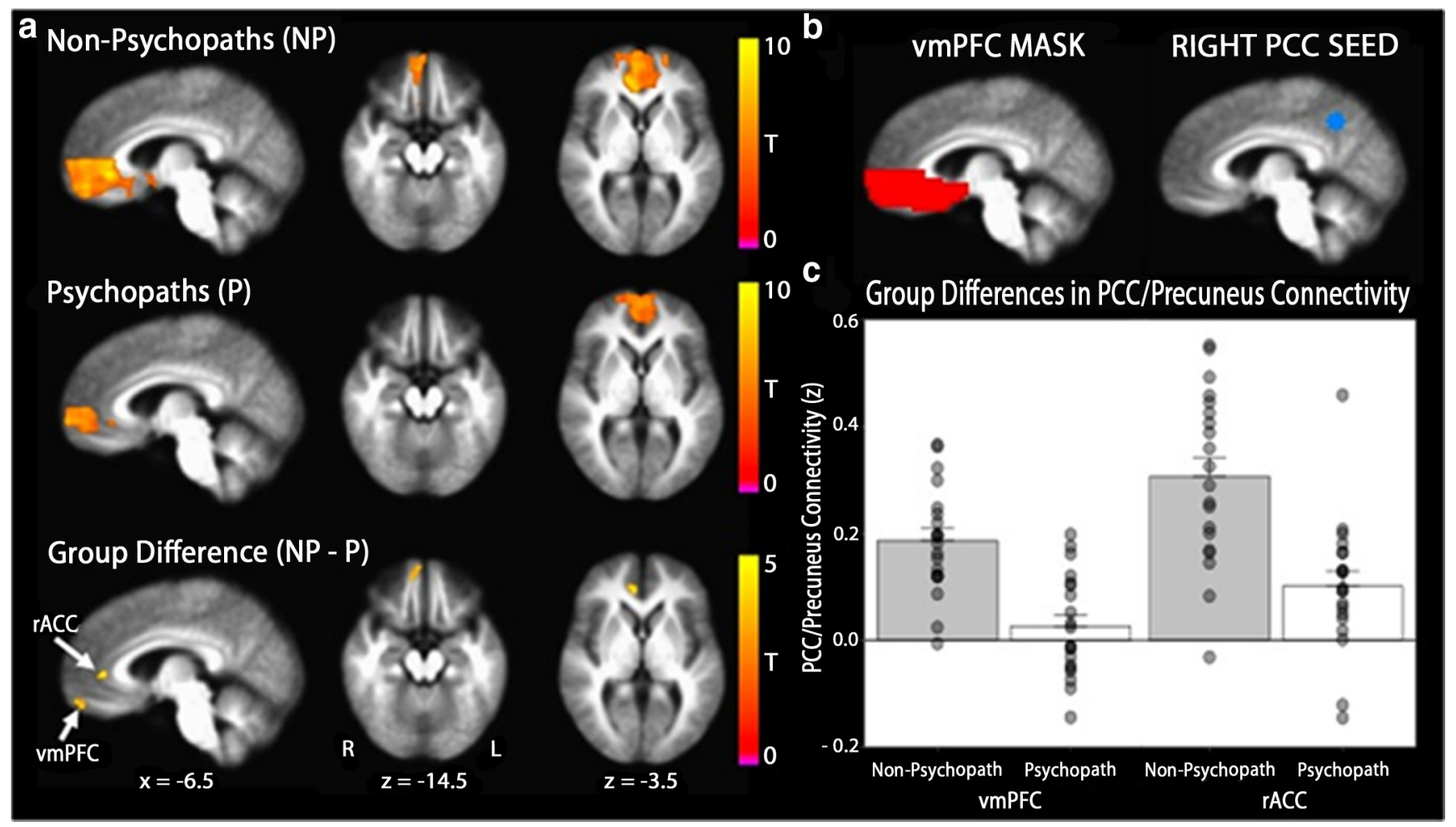

Figure 3. Functional connectivity between medial parietal cortex and vmPFC is reduced in psychopaths. $\boldsymbol{a}$, Mean precuneus/PCC-vmPFC connectivity maps are depicted separately for nonpsychopaths and psychopaths on the group mean anatomical image, thresholded at a cluster corrected $p<0.05$. Scale bars indicate the uncorrected $t$ statistic. Both groups exhibit significant resting connectivity between right precuneus/PCC and regions of vmPFC. The group difference map indicates two separate clusters within vmPFC where non-psychopaths have significantly greater connectivity with the precuneus/PCC seed than psychopaths (vmPFC: $x=-12, y=-51, z=-12$, cluster size $=27$ voxels; rACC: $x=-9, y=-39, z=+6$, cluster size $=15$ voxels). $\boldsymbol{b}$, Group differences in connectivity were assessed in the $\operatorname{VmPFC}$ mask (red) for correlation coefficients computed using the mean time series extracted from the PCC seed (blue; $x=-5, y=+49, z=+40)$. c, The bar plots depict group differences in connectivity estimates (Fisher z-transformed correlation coefficients) within each significant cluster. Error bars indicate SEM. Filled circles represent values from individual subjects.

To address the anatomical specificity of this finding, we assessed resting connectivity between the precuneus/PCC seed region and two additional areas within the default mode network that have been shown to be functionally connected with precuneus/PCC at rest: the IPL and the parahippocampal gyrus (Greicius et al., 2003; Fox et al., 2005). Although both psychopaths and non-psychopaths had significant positive connectivity between each area and the precuneus/PCC, in neither ipsilateral area were there significant differences in connectivity between groups. In other words, psychopaths exhibited normal functional connectivity between right precuneus/PCC and right IPL, as well as between right precuneus/PCC and right parahippocampal gyrus. By contrast, psychopaths exhibited abnormally low levels of functional connectivity between right precuneus/PCC and vmPFC.

In sum, these fMRI data associate psychopathy with a reduction of resting functional connectivity in circuits involving vmPFC.

Follow-up analyses: psychopathy subtypes

For each of the three main findings associated with psychopathy in this study (reduced FA in right UF, reduced resting vmPFC BOLD signal correlation with amygdala, and reduced resting vmPFC BOLD signal correlation with precuneus/PCC), we next considered whether each finding was driven by a particular subtype of psychopathy (low-anxious/"primary" vs high-anxious/ "secondary").

First we examined the DTI finding of reduced FA in the right UF among psychopaths. Because of the relatively small numbers of inmates with primary and secondary psychopathy in the DTI sample ( $n=7$ and $n=7$, respectively), we used non-parametric Mann-Whitney $U$ tests to assess differences between subtypes. As can be seen in Figure $4 b$, the high-anxious non-psychopaths exhibited significantly lower right UF FA values than the lowanxious non-psychopaths ( $p=0.046$ ), whereas the high-anxious psychopaths and low-anxious psychopaths did not significantly differ $(p=0.75)$.

Next we considered the rest-fMRI finding of lower vmPFCamygdala BOLD signal correlation among psychopaths. Given the slightly larger number of inmates with primary and secondary psychopathy in the rest-fMRI sample $(n=12$ and $n=8$, respectively), we conducted a $2 \times 2$ (psychopathy: high/low $\times$ anxiety high/low) ANOVA. Here we found a significant interaction between psychopathy and anxiety $(F=9.1, p=0.005)$, such that higher anxiety was associated with greater connectivity among non-psychopaths, but lower connectivity among psychopaths (Fig. 4c). Post hoc $t$ tests revealed no significant difference in connectivity between high- and low-anxious non-psychopaths $(t=1.7, p=0.12)$, but significantly greater connectivity in lowanxious ("primary") psychopaths than high-anxious ("secondary") psychopaths $(t=2.6, p=0.02)$. Thus, in addition to discriminating between psychopaths and non-psychopaths, amygdala-vmPFC connectivity distinguishes between psychopathy subtypes.

Finally we considered the rest-fMRI finding of lower vmPFCprecuneus/PCC BOLD signal correlation among psychopaths. Here we found no significant interaction between psychopathy 


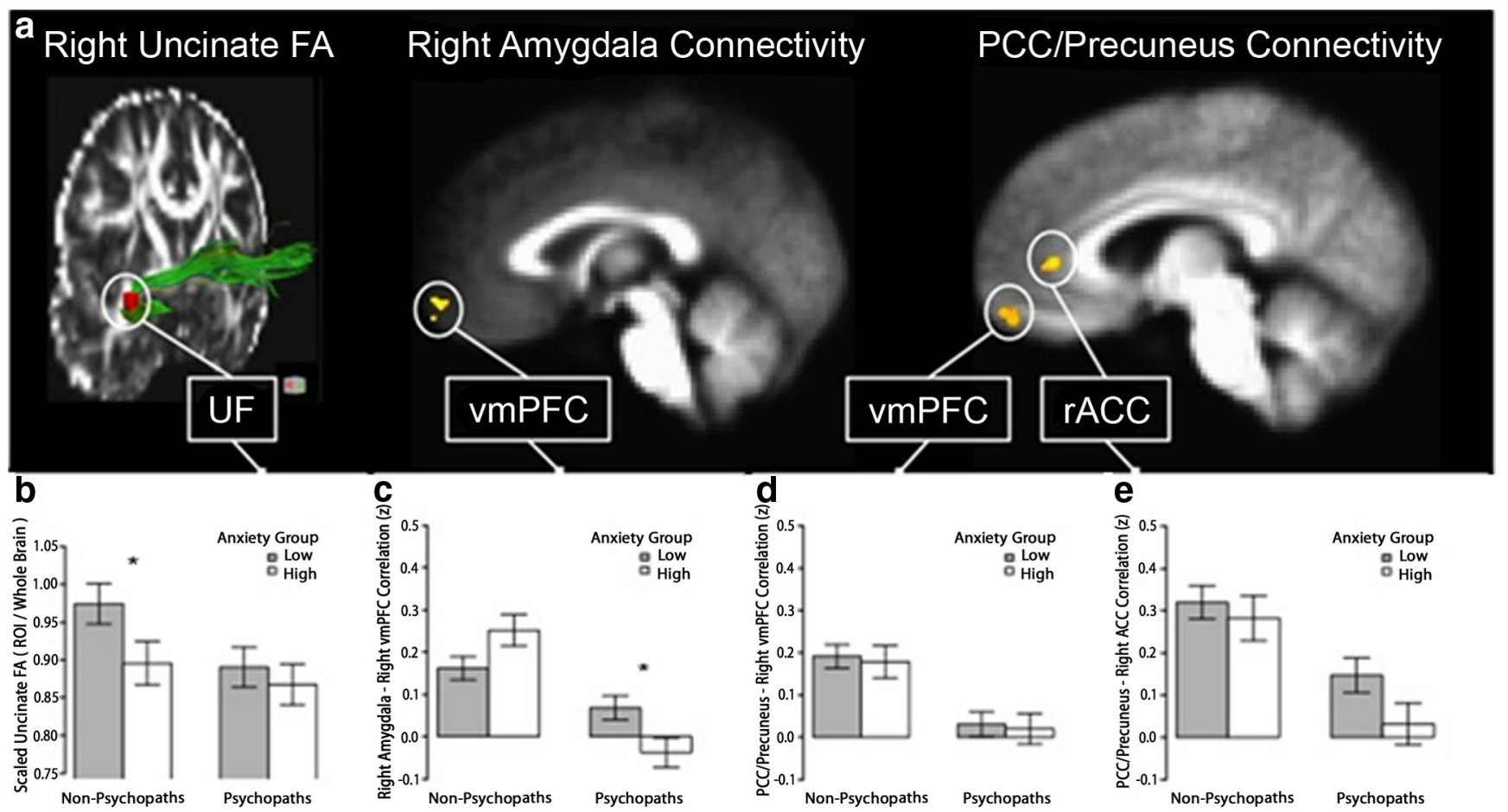

Figure 4. Analysis of DTI and rest-fMRI findings with respect to psychopathy subtype. $\boldsymbol{a}$, Depiction of each significant finding in the main between-group analyses (psychopaths vs nonpsychopaths). $\boldsymbol{b}$, For right UF FA values, among non-psychopaths, the low- and high-anxious subgroups significantly differ ( $p=0.046)$; there is no such difference among psychopaths. c, For the cluster identified in the right amygdala-vmPFC connectivity analysis, there is a significant interaction between psychopathy and anxiety $(p=0.005)$, indicating that connectivity is differentially modulated by anxiety subgroup in psychopaths and non-psychopaths. $\boldsymbol{d}, \boldsymbol{e}$, There are no significant interactions between psychopathy and anxiety for medial parietal-vmPFC connectivity. Error bars indicate SEM. Asterisks indicate significant differences between anxiety subtypes.

and anxiety for either the anterior vmPFC area $(F=0.002, p=$ 0.97) (Fig. $4 d)$ or the rACC area $(F=0.73, p=0.40)$ (Fig. $4 e$ ).

In sum, consideration of psychopathy subtype (low-anxious vs high-anxious) yielded significant effects for UF structural integrity and vmPFC-amygdala functional connectivity, but not for vmPFC-medial parietal functional connectivity.

\section{Discussion}

In this study of psychopathic and non-psychopathic prison inmates, we found evidence of significantly reduced vmPFC connectivity among psychopaths: lower FA in right UF and lower BOLD signal correlations with amygdala and precuneus/PCC at rest. In addition, we found preliminary evidence that the structural and functional differences in vmPFC-amygdala connectivity between psychopaths and non-psychopaths may vary as a function of psychopathic subtype. In the following sections, we discuss each of these findings in turn.

\section{Structural connectivity: reduced FA in right UF}

Ours is not the first study to use DTI to assess white matter structural integrity in psychopathy. The only previous study to do so also found that individuals with higher psychopathy scores had significantly lower FA values in right UF than those with lower psychopathy scores (Craig et al., 2009). Although the data presented here support the conclusion of the previous study (reduced structural connectivity between vmPFC and amygdala in psychopathy), our study has several notable methodological differences.

One advantage is a more rigorous subject classification scheme. The previous study included $n=9$ adult males with relatively high levels of psychopathy, defined as PCL-R score $\geq 25$ (mean $=28.4)$. We used a more stringent PCL-R cutoff of $\geq 30$ to define our psychopathic group, in accordance with the PCL-R recommendations (Hare, 2003) and in recognition of the fact that individuals with intermediate PCL-R scores (21-29) are in some experimental paradigms more similar to non-psychopaths (PCL-R $\leq 20$ ) than actual psychopaths (PCL-R $\geq 30$ ) [e.g., in tests of emotion-modulated startle (Patrick et al., 1993) and moral judgment (Koenigs et al., 2011b)]. In addition, our psychopathic and non-psychopathic groups were well matched on demographic variables, intelligence, and substance abuse histories (Table 1).

A second distinguishing feature of the DTI component of our study is the single ROI-based approach to calculate UF FA values. The ROI we used was within the dense insular segment, or "isthmus," of the UF. This ROI excludes terminal regions of the UF that intermingle with adjacent tracts (Ebeling and von Cramon, 1992). Combining this ROI approach with the correction for whole-brain FA differences allowed us to isolate regions of particular interest, while accounting for non-specific global differences throughout the brain.

Finally, due to a larger sample of psychopaths $(n=14)$, we were able to address the role of psychopathic subtypes in mediating the observed effect (see "Psychopathic subtypes" below for detailed discussion of this point).

Despite the methodological differences between studies, the fact that both DTI studies reveal the same specific finding supports a robust association between psychopathy and reduced FA in right $U F$.

Functional connectivity: reduced vmPFC-amygdala correlation at rest

Although several previous $\mathrm{fMRI}$ studies have associated psychopathy with abnormal activation of the amygdala and vmPFC (see 
Koenigs et al., 2011a for review), our study is the first to demonstrate aberrant functional connectivity between vmPFC and amygdala in psychopathy using rest-fMRI. Moreover, the collection of neuroimaging data from $n=20$ psychopaths with PCL-R scores $\geq 30$ is the largest such sample ever reported in an MRI study.

The finding of reduced vmPFC-amygdala correlation at rest coincides with our structural DTI data; both findings indicate diminished connectivity between vmPFC and amygdala in psychopathy. These convergent results support the central hypothesis of this study, and join a substantial corpus of research highlighting the importance of vmPFC-amygdala interactions in the regulation of emotion and social behavior (Barbas, 2000; Davidson, 2002; Milad et al., 2006; Delgado et al., 2008). Additionally, these neuroimaging findings are consistent with the longstanding psychological perspective that impaired decision making in psychopathy is due to deficient integration of affective information (Cleckley, 1976; Hiatt and Newman, 2006; Blair and Mitchell, 2009). The amygdala is understood to signal the affective salience of environmental stimuli (Davis and Whalen, 2001; Adolphs, 2010), and the anterior vmPFC is purported to discriminate the relative value of options and outcomes during decision making (O’Doherty, 2004; Grabenhorst and Rolls, 2011). Accordingly, our finding of reduced functional connectivity between these regions suggests a plausible neurobiological mechanism underlying abnormal decision making in psychopathy. More generally, the convergent DTI and rest-fMRI results suggest that the socio-affective deficits characterizing psychopathy may reflect impaired communication between vmPFC and amygdala.

This conclusion is supported by a previous study of adolescents with callous and unemotional traits, which revealed reduced functional connectivity between the amygdala and vmPFC during facial affect processing (Marsh et al., 2008). The convergence of findings across distinct demographic samples and experimental contexts suggests that reduced amygdala-vmPFC connectivity may be a consistent neurobiological feature of populations in which callous unemotionality and impaired empathy are major characteristics.

A goal for future research will be to use task-related fMRI to examine whether reduced vmPFC-amygdala connectivity is an invariant feature of the psychopathic brain, or if this abnormality is diminished or exaggerated during certain cognitive or affective tasks. Additionally, it will be important to investigate with more anatomically precise imaging techniques whether the connectivity differences we observe in psychopaths are driven by particular subnuclei of the amygdala.

\section{Functional connectivity: reduced vmPFC-precuneus/PCC correlation at rest}

The second major rest-fMRI finding of this study is reduced connectivity between vmPFC and precuneus/PCC. Again, ours is the first study to demonstrate this effect. The vmPFC and precuneus/ PCC comprise two nodes of the default mode network (Raichle et al., 2001; Greicius et al., 2003). It is important to note that the reduced correlation between vmPFC and precuneus/PCC activity among psychopaths does not simply reflect diminished functional connectivity throughout the default mode network. Functional connectivity was normal between right PCC/precuneus and two other nodes of the network: right inferior parietal lobule and right parahippocampal gyrus.

Although the functional significance of the vmPFC-precuneus/PCC circuit remains to be fully elucidated, preliminary ac- counts suggest involvement in self-reflective cognition (Buckner et al., 2008; Qin and Northoff, 2011). Previous empirical and theoretical work on psychopathy has proposed an underlying defect in self-reflection, particularly as it pertains to observed decision-making impairments (Newman and Lorenz, 2003; Koenigs et al., 2010). However, at this point it may be premature to speculate on the precise functional significance of reduced vmPFC-medial parietal connectivity in psychopathy. For the primary aim of this study (identifying neural correlates of psychopathy), the default network provides a well validated means of testing the functional connectivity of vmPFC. As with the amygdala seed, the precuneus/PCC seed reveals diminished functional connectivity of vmPFC in the psychopathic brain.

\section{Psychopathic subtypes}

This is the first neuroimaging study to directly test whether the neural correlates of psychopathy vary as a function of psychopathic subtype. In light of the decades of theoretical and empirical work emphasizing a distinction between low-anxious ("primary") and high-anxious ("secondary") psychopathic subtypes (e.g., Karpman, 1946; Lykken, 1957; Blackburn, 1975; Cleckley, 1976; Newman et al., 1990, 1992; Hiatt et al., 2004; Koenigs et al., 2010), we evaluated whether trait anxiety/negative affect among psychopaths has any significant bearing on the observed neurobiological differences between psychopaths and non-psychopaths.

With respect to the DTI finding of reduced FA in right UF, the effect appears to be driven primarily by a difference between low-anxious psychopaths and low-anxious non-psychopaths. Among non-psychopaths, we observed the expected relationship between UF and anxiety; namely, lower levels of anxiety were associated with greater structural integrity of the UF (Kim and Whalen, 2009), presumably due to more efficient regulation of emotion through greater PFC-amygdala connectivity (Davidson, 2002; Milad et al., 2006). Psychopaths, on the other hand, did not exhibit this relationship between UF integrity and anxiety. We speculate that the low levels of anxiety in primary psychopathy may be mediated by a different neurocognitive mechanism than efficient "top-down" regulation of emotion through a PFC-amygdala circuit. Perhaps the low anxiety (and overall emotional unresponsiveness) of primary psychopaths is driven instead by deficient "bottom-up" integration of affective information (Hiatt and Newman, 2006; Blair and Mitchell, 2009; Baskin-Sommers et al., 2011).

With respect to the rest-fMRI findings of less correlated activity in vmPFC circuits among psychopaths, we saw a significant interaction between psychopathy and anxiety for vmPFC-amygdala connectivity, but no significant interaction between psychopathy and anxiety for the vmPFC-precuneus/PCC connectivity. Thus, the significant effect of psychopathic subtype is restricted to structural and functional connectivity between vmPFC and amygdala. In particular, we note that vmPFC-amygdala functional connectivity not only distinguishes psychopaths from non-psychopaths, but also primary (low-anxious) psychopaths from secondary (high-anxious) psychopaths. Although this finding merits attention as the first evidence for dissociable neural substrates of psychopathic subtypes, the functional significance of these subtype data is not immediately clear. Future work with larger samples and tasks specifically designed to elicit vmPFC-amygdala interactions (e.g., reversal learning or emotion regulation) will be necessary to better understand the effect of psychopathy subtype on amygdala-vmPFC connectivity.

We believe that the present data, while preliminary, indicate that consideration of psychopathic subtypes may be of critical 
importance for clarifying the neural correlates of psychopathy. Further research will be necessary to elucidate whether, within the neurobiological characteristics that broadly distinguish psychopaths from non-psychopaths, there are also features that reliably distinguish between psychopathic subtypes.

\section{Limitations}

A limitation of the extreme groups design used in this study is that we are unable to determine whether our findings are driven by a particular factor of psychopathy (interpersonal-affective or impulsive-antisocial) or by particular behavioral characteristics (e.g., aggression). Future work in populations with more continuous distributions of psychopathy scores will be necessary to assess whether a particular dimensional factor drives the effects we report here.

\section{Conclusion}

Collectively, the structural and functional neuroimaging data presented here converge to specify diminished vmPFC connectivity as a robust neural correlate of psychopathy. Human lesion studies have long implicated vmPFC dysfunction in the pathogenesis of "psychopathic" behavioral and affective traits (Eslinger and Damasio, 1985; Damasio et al., 1990; Koenigs et al., 2010). The imaging findings presented here provide direct evidence of vmPFC dysfunction in criminal psychopaths, and putatively identify a specific neurobiological abnormality underlying psychopathy.

\section{References}

Adolphs R (2010) What does the amygdala contribute to social cognition? Ann N Y Acad Sci 1191:42-61.

Alexander DC, Barker GJ (2005) Optimal imaging parameters for fiberorientation estimation in diffusion MRI. Neuroimage 27:357-367.

Andersson JLR, Jenkinson M, Smith S (2007) Non-linear registration, aka spatial normalisation. FMRIB technical report TR07JA2.

Arnett PA, Smith SS, Newman JP (1997) Approach and avoidance motivation in psychopathic criminal offenders during passive avoidance. J Pers Soc Psychol 72:1413-1428.

Barbas H (2000) Connections underlying the synthesis of cognition, memory, and emotion in primate prefrontal cortices. Brain Res Bull 52:319-330.

Baskin-Sommers AR, Curtin JJ, Newman JP (2011) Specifying the attentional selection that moderates the fearlessness of psychopathic offenders. Psychol Sci 22:226-234.

Blackburn R (1975) An empirical classification of psychopathic personality. Br J Psychiatry 127:456-460.

Blair RJ (2007) The amygdala and ventromedial prefrontal cortex in morality and psychopathy. Trends Cogn Sci 11:387-392.

Blair RJ (2008) The amygdala and ventromedial prefrontal cortex: functional contributions and dysfunction in psychopathy. Philos Trans R Soc Lond B Biol Sci 363:2557-2565.

Blair RJ, Mitchell DG (2009) Psychopathy, attention and emotion. Psychol Med 39:543-555.

Blumer D, Benson DF (1975) Personality changes with frontal and temporal lesions. In: Psychiatric aspects of neurological disease (Benson DF, Blumer D, eds). New York: Stratton.

Brinkley CA, Newman JP, Widiger TA, Lyman DR (2004) Two approaches to parsing the heterogeneity of psychopathy. Clin Psychol Sci Practice 11:69-94.

Buckner RL, Andrews-Hanna JR, Schacter DL (2008) The brain's default network: anatomy, function, and relevance to disease. Ann N Y Acad Sci 1124:1-38.

Cleckley H (1976) The mask of sanity, Ed 5. St. Louis: Mosby.

Cook PA, Bai Y, Nedjati-Gilani S, Seunarine KK, Hall MG, Parker GJ (2006) CAMINO: open-source diffusion-MRI reconstruction and processing. 14th Scientific Meeting of the International Society for Magnetic Resonance in Medicine. Seattle.
Cox RW (1996) AFNI: software for analysis and visualization of functional magnetic resonance neuroimages. Comput Biomed Res 29:162-173.

Craig MC, Catani M, Deeley Q, Latham R, Daly E, Kanaan R, Picchioni M, McGuire PK, Fahy T, Murphy DG (2009) Altered connections on the road to psychopathy. Mol Psychiatry 14:946-953, 907.

Damasio AR, Tranel D, Damasio H (1990) Individuals with sociopathic behavior caused by frontal damage fail to respond autonomically to social stimuli. Behav Brain Res 41:81-94.

Davidson RJ (2002) Anxiety and affective style: role of prefrontal cortex and amygdala. Biol Psychiatry 51:68-80.

Davidson RJ, Putnam KM, Larson CL (2000) Dysfunction in the neural circuitry of emotion regulation-a possible prelude to violence. Science 289:591-594.

Davis M, Whalen PJ (2001) The amygdala: vigilance and emotion. Mol Psychiatry 6:13-34.

Delgado MR, Nearing KI, Ledoux JE, Phelps EA (2008) Neural circuitry underlying the regulation of conditioned fear and its relation to extinction. Neuron 59:829-838.

Ebeling U, von Cramon D (1992) Topography of the uncinate fascicle and adjacent temporal fiber tracts. Acta Neurochir (Wien) 115:143-148.

Eslinger PJ, Damasio AR (1985) Severe disturbance of higher cognition after bilateral frontal lobe ablation: patient EVR. Neurology 35:1731-1741.

First MB, ed (2002) Structured Clinical Interview for DSM-IV-TR Axis I Disorders, Research Version, Non-patient Edition. (SCID-I/NP). New York: Biometrics Research, New York State Psychiatric Institute.

Fox MD, Snyder AZ, Vincent JL, Corbetta M, Van Essen DC, Raichle ME (2005) The human brain is intrinsically organized into dynamic, anticorrelated functional networks. Proc Natl Acad Sci U S A 102:9673-9678.

Glenn AL, Raine A, Schug RA (2009) The neural correlates of moral decision-making in psychopathy. Mol Psychiatry 14:5-6.

Grabenhorst F, Rolls ET (2011) Value, pleasure and choice in the ventral prefrontal cortex. Trends Cogn Sci 15:56-67.

Greicius MD, Krasnow B, Reiss AL, Menon V (2003) Functional connectivity in the resting brain: a network analysis of the default mode hypothesis. Proc Natl Acad Sci U S A 100:253-258

Hare RD (2003) The Hare psychopathy checklist-revised, Ed 2. Toronto: Multi-Health Systems.

Hiatt KD, Newman JP (2006) Understanding psychopathy: the cognitive side. In: Handbook of psychopathy (Patrick CJ, ed), pp 334-352. New York: Guilford.

Hiatt KD, Schmitt WA, Newman JP (2004) Stroop tasks reveal abnormal selective attention among psychopathic offenders. Neuropsychology 18:50-59.

Hua K, Zhang J, Wakana S, Jiang H, Li X, Reich DS, Calabresi PA, Pekar JJ, van Zijl PC, Mori S (2008) Tract probability maps in stereotaxic spaces: analyses of white matter anatomy and tract-specific quantification. Neuroimage 39:336-347.

Jones DK, Basser PJ (2004) "Squashing peanuts and smashing pumpkins": how noise distorts diffusion-weighted MR data. Magn Reson Med 52:979-993.

Karpman B (1946) Psychopathy in the scheme of human typology. J Nerv Ment Dis 103:276-288.

Kiehl KA (2006) A cognitive neuroscience perspective on psychopathy: evidence for paralimbic system dysfunction. Psychiatry Res 142:107-128.

Kiehl KA, Smith AM, Hare RD, Mendrek A, Forster BB, Brink J, Liddle PF (2001) Limbic abnormalities in affective processing by criminal psychopaths as revealed by functional magnetic resonance imaging. Biol Psychiatry 50:677-684.

Kim MJ, Whalen PJ (2009) The structural integrity of an amygdalaprefrontal pathway predicts trait anxiety. J Neurosci 29:11614-11618.

Kim MJ, Gee DG, Loucks RA, Davis FC, Whalen PJ (2011) Anxiety dissociates dorsal and ventral medial prefrontal cortex functional connectivity with the amygdala at rest. Cereb Cortex 21:1667-1673.

Koenigs M, Kruepke M, Newman JP (2010) Economic decision-making in psychopathy: a comparison with ventromedial prefrontal lesion patients. Neuropsychologia 48:2198-2204.

Koenigs M, Baskin-Sommers A, Zeier J, Newman JP (2011a) Investigating the neural correlates of psychopathy: a critical review. Mol Psychiatry 16:792-799.

Koenigs M, Kruepke M, Zeier J, Newman JP (2011b) Utilitarian moral judgment in psychopathy. Soc Cogn Affect Neurosci. Advance online publication. doi:10.1093/scan/nsr048. 
Lazar M, Weinstein DM, Tsuruda JS, Hasan KM, Arfanakis K, Meyerand ME, Badie B, Rowley HA, Haughton V, Field A, Alexander AL (2003) White matter tractography using diffusion tensor deflection. Hum Brain Mapp 18:306-321.

Lorenz AR, Newman JP (2002) Deficient response modulation and emotion processing in low-anxious Caucasian psychopathic offenders: results from a lexical decision task. Emotion 2:91-104.

Lykken DT (1957) A study of anxiety in the sociopathic personality. J Abnorm Psychol 55:6-10.

Maldjian JA, Laurienti PJ, Kraft RA, Burdette JH (2003) An automated method for neuroanatomic and cytoarchitectonic atlas-based interrogation of fMRI data sets. Neuroimage 19:1233-1239.

Marsh AA, Finger EC, Mitchell DG, Reid ME, Sims C, Kosson DS, Towbin KE, Leibenluft E, Pine DS, Blair RJ (2008) Reduced amygdala response to fearful expressions in children and adolescents with callousunemotional traits and disruptive behavior disorders. Am J Psychiatry 165:712-720.

Milad MR, Rauch SL, Pitman RK, Quirk GJ (2006) Fear extinction in rats: implications for human brain imaging and anxiety disorders. Biol Psychol 73:61-71.

Mori S, Wakana S, Nagae-Poetscher L, van Zijl PCM (2005) MRI atlas of human white matter. Amsterdam: Elsevier.

Nacewicz BM, Dalton KM, Johnstone T, Long MT, McAuliff EM, Oakes TR, Alexander AL, Davidson RJ (2006) Amygdala volume and nonverbal social impairment in adolescent and adult males with autism. Arch Gen Psychiatry 63:1417-1428.

Newman JP, Lorenz AR (2003) Response modulation and emotion processing: implications for psychopathy and other dysregulatory psychopathology. In: Handbook of affective sciences (Davidson RJ, Scherer K, Goldsmith HH, eds), pp 904-929. Oxford: Oxford UP.

Newman JP, Patterson CM, Howland EW, Nichols SL (1990) Passive avoidance in psychopaths: the effects of reward. Pers Individ Dif 11:1101-1114.

Newman JP, Kosson DS, Patterson CM (1992) Delay of gratification in psychopathic and nonpsychopathic offenders. J Abnorm Psychol 101:630-636.

O’Doherty JP (2004) Reward representations and reward-related learning in the human brain: insights from neuroimaging. Curr Opin Neurobiol 14:769-776.
Patrick CJ, Bradley MM, Lang PJ (1993) Emotion in the criminal psychopath: startle reflex modulation. J Abnorm Psychol 102:82-92.

Pierpaoli C, Basser PJ (1996) Toward a quantitative assessment of diffusion anisotropy. Magn Reson Med 36:893-906.

Price JL (1999) Prefrontal cortical networks related to visceral function and mood. Ann N Y Acad Sci 877:383-396.

Qin P, Northoff G (2011) How is our self related to midline regions and the default-mode network? Neuroimage 57:1221-1233.

Raichle ME, MacLeod AM, Snyder AZ, Powers WJ, Gusnard DA, Shulman GL (2001) A default mode of brain function. Proc Natl Acad Sci U S A 98:676-682.

Roy AK, Shehzad Z, Margulies DS, Kelly AM, Uddin LQ, Gotimer K, Biswal BB, Castellanos FX, Milham MP (2009) Functional connectivity of the human amygdala using resting state fMRI. Neuroimage 45:614-626.

Shulman GL, Fiez JA, Corbetta M, Buckner RL, Miezin FM, Raichle ME, et al. (1997) Common blood flow changes across visual tasks: II. Decreases in cerebral cortex. J Cogn Neurosci 9:648-663.

Smith SM (2002) Fast robust automated brain extraction. Hum Brain Mapp 17:143-155.

Talairach J, Tournoux P (1988) Co-planar stereotaxic atlas of the human brain. New York: Thieme Medical.

Wakana S, Caprihan A, Panzenboeck MM, Fallon JH, Perry M, Gollub RL, Hua K, Zhang J, Jiang H, Dubey P, Blitz A, van Zijl P, Mori S (2007) Reproducibility of quantitative tractography methods applied to cerebral white matter. Neuroimage 36:630-644.

Wang R, Benner T, Sorensen AG, Wedeen VJ (2007) Diffusion toolkit: a software package for diffusion imaging data processing and tractography. Proc Int Soc Mag Reson Med 15:3720.

Welsh G (1956) Factor dimensions A and R. In: Basic readings on the MMPI in psychology and medicine (Welsh GS, Dahlstrom WG, eds), pp 264281. Minneapolis: University of Minnesota Press.

Zachary RA (1986) Shipley Institute of Living Scale: Revised Manual. Los Angeles: Western Psychological Services.

Zhang Y, Brady M, Smith S (2001) Segmentation of brain MR images through a hidden Markov random field model and the expectationmaximization algorithm. IEEE Trans Med Imaging 20:45-57. 